\title{
Heart Rhythm Society Members' Views on Pacemaker and Implantable Cardioverter-Defibrillator Reuse
}

\author{
ANDREW B. HUGHEY, M.D., * NIMIT DESAI, B.S., * TIMIR S. BAMAN, M.D.,† \\ LINDSEY GAKENHEIMER, B.S., * LINDSAY HAGAN, B.S., * JAMES N. KIRKPATRICK, M.D., $*$ \\ HAKAN ORAL, M.D., * KIM A. EAGLE, M.D., * and THOMAS C. CRAWFORD, M.D.* \\ From the *University of Michigan Cardiovascular Center, Ann Arbor, Michigan; +Department of Medicine, \\ University of Illinois College of Medicine at Peoria, Peoria, Illinois; and ¥Cardiovascular Medicine Division, \\ University of Pennsylvania, Philadelphia, Pennsylvania
}

Background: Reuse of cardiac implantable electronic devices (CIEDs) may help address the unmet need among patients in low- and middle-income countries (LMICs).

Methods: To examine Heart Rhythm Society (HRS) physicians' opinions regarding CIED reuse, an online survey eliciting attitudes toward CIED reuse was sent to all 3,380 HRS physician members.

Results: There were 429 responses (response rate 13\%). A large majority of respondents agreed or strongly agreed that resterilization of devices for reimplantation in patients who cannot afford new devices may be safe $(370,87 \%)$ and, if proven to be safe, would be ethical $(375,88 \%)$. A total of $340(81 \%)$ respondents would be comfortable asking their patients to consider donating their device, and 353 (84\%) would be willing to reimplant a resterilized device if it were legal. The most commonly cited concerns about device reuse were infection $(270,64 \%)$ and device malfunction (125, 29\%). Respondents from the United States and Canada had more favorable impressions of device reuse than respondents from other high-income countries ( $P<0.05$ for three of five positive statements regarding reuse), and were less likely to cite ethical concerns $(P<0.001)$. However, when responses from all high-income countries were compared with lower- and upper-middle income countries, there were no significant differences in the rates of approval.

Conclusions: HRS survey respondents support the concept of CIED reuse for patients in LMICs who cannot afford new devices. Studies are needed to demonstrate the clinical efficacy and safety of this practice and to identify potential barriers to adoption among physicians. (PACE 2014; 37:969-977)

pacing, defibrillation-ICD, reutilization, reuse, views, opinions

\section{Introduction}

Pacemakers and implantable cardioverter defibrillators (ICDs) have revolutionized the treatment of bradyarrythmias and tachyarrythmias in wealthy countries, but unfortunately they remain unaffordable for many patients in low- and middle-income countries (LMICs). ${ }^{1}$ The reuse of pacemakers and ICDs, which involves harvesting devices from deceased patients, device selection and sterilization, and implantation in recipient patients, may help those with no access to this therapy in underserved nations. Numerous small

Disclosures: My Heart-Your Heart initiative is supported by grants from the Hewlett Foundation, the Mardigian Foundation, the University of Michigan Cardiovascular Center, and gifts from Mr. and Mrs. Sheldon Davis and Mr. and Mrs. Craig Sincock. There are no conflicts of interest.

Address for reprints: Thomas C. Crawford, M.D., 1500 East Medical Center Drive SPC 5853, Ann Arbor, MI 48109-5853. Fax: 734-936-7026; e-mail: thomcraw@med.umich.edu

Received December 8, 2013; revised March 5, 2014; accepted March 18, 2014.

doi: 10.1111/pace.12418 studies in a variety of settings worldwide have demonstrated the feasibility, efficacy, and safety of pacemaker and ICD reuse (Appendix S1 in the Supporting information).

A successful large-scale device reuse program would necessitate the coordinated participation of many stakeholders. As the majority of devices available for reuse are harvested from deceased patients, potential donor patients and their families, funeral home and crematory directors, as well as physicians and allied health professionals, all have a role to play. Project My Heart-Your Heart is a collaboration between the University of Michigan Cardiovascular Center, World Medical Relief, the Michigan Funeral Home Director Association, Implant Recycling, Inc., and numerous funeral homes and crematories throughout the United States. Project My HeartYour Heart seeks to facilitate pacemaker reuse in order to benefit individuals in LMICs. ${ }^{2}$ Previously published survey data indicate that $89 \%$ of funeral directors, $87 \%$ of patients with implantable devices, and $71 \%$ of the general population would support a pacemaker reutilization initiative if given the opportunity. ${ }^{3}$ However, little is

(C)2014 Wiley Periodicals, Inc. 
currently known about Heart Rhythm Society (HRS) physician members' opinions regarding device reuse. Physician acceptance is essential to the success of a device reutilization program, including those in donor countries (to facilitate device explantation, screening for reusability, and sterilization) as well as those in recipient countries (to select appropriate recipient patients and perform reimplantation). In this study, we aimed to quantitatively evaluate the attitudes of HRS physician members toward cardiac implantable electronic device (CIED) reuse, and to identify potential barriers to adoption.

\section{Methods}

\section{Survey}

We developed an online survey to elicit attitudes toward CIED reuse. The survey instrument was approved by Membership and Executive committees of the HRS and sent electronically using SurveyMonkey (SurveyMonkey, Palo Alto, CA, USA) to each of the 3,380 physician members of the HRS. The survey consisted of 29 questions (Appendix S2 in the Supporting information). Demographic questions included physician specialty, the type of practice institution (university/academic vs private practice, etc.), practice setting (urban vs suburban vs rural), practice country, and country of birth. Respondents were asked to rate their level of agreement with various positive statements regarding device reuse using a Likert scale $(1=$ strongly disagree, $2=$ disagree, $3=$ neutral, $4=$ agree, and $5=$ strongly agree). Respondents were also asked to identify, in multiple-choice format, their greatest concern(s) regarding device reuse.

\section{Data Analysis}

To simplify the analysis, responses of "disagree" and "strongly disagree" were tabulated and analyzed together, and responses of "agree" and "strongly agree" were tabulated and analyzed together.

In addition to seeking the attitudes of survey respondents in aggregate, we also hypothesized that respondents from poorer countries would have more positive views regarding CIED reuse than those from wealthier countries, and that respondents from the United States and Canada may have different views from those in other highincome countries. Respondents were therefore grouped into one of three categories based on their country of practice: (1) the United States and Canada, (2) high-income countries excluding the United States and Canada, and (3) lowerand upper-middle income countries. High-income countries were defined as those designated by the World Bank as having "high-income economies" (per capita income of $\$ 12,616$ USD or greater)this group included respondents from Australia, Austria, Belgium, Finland, Germany, Greece, Ireland, Israel, Italy, Japan, South Korea, Monaco, Netherlands, New Zealand, Poland, Saudi Arabia, Singapore, Spain, Sweden, United Arab Emirates, and the United Kingdom. Lower- and uppermiddle income countries were similarly defined as those designated by the World Bank as having "lower-middle income economies" (per capita income of $\$ 1,036$ to $\$ 4,085$ USD) or "upper-middle income economies" (per capita income of $\$ 4,086$ to $\$ 12,615$ USD) - this group included respondents from Argentina, Belize, Brazil, China, Colombia, India, Iran, Malaysia, Mexico, Pakistan, Peru, Philippines, Romania, Sri Lanka, and Taiwan. ${ }^{4}$ There were no respondents from World Bank "low-income" countries (per capita income of $\$ 1,035$ USD or less). Using the $\chi^{2}$ test, responses were compared across the three income economy groups represented (i.e., United States and Canada vs other highincome countries vs lower- and upper-middle income countries). A simpler two-group analysis was also performed that compared responses from all high-income countries (United States, Canada, and others) against those from lower- and upper-middle income countries. Analyses were performed using SPSS for Windows (IBM Corp., Armonk, NY, USA).

\section{Results}

\section{Respondent Characteristics and General Perceptions}

There were 429 responses (response rate $13 \%)$. The characteristics of the respondents are shown in Table I. The majority were electrophysiologists $(N=389,91 \%)$, followed by general cardiologists $(N=15,3.5 \%)$ and pediatric cardiologists $(N=8,1.9 \%)$. Two hundred sixteen $(50 \%)$ operated at a university or academic medical center and about one quarter worked in private medical practice $(N=115,27 \%)$. The respondents' practice settings were primarily urban $(N=301,70 \%)$. The majority $(N=298$, $70 \%$ ) of respondents practiced in the United States, although only $210(50.1 \%)$ had been born in the United States. Thirty-nine respondents $(9.2 \%)$ were from lower- or upper-middle income countries.

Three hundred ninety-nine (93\%) respondents had implanted a cardiac device in the preceding 12 months, with $353(82 \%)$ reporting implanting greater than 25 pacemakers per year, and $309(72 \%)$ reporting implanting greater than 25 ICDs or biventricular ICDs. Three hundred fiftysix $(84 \%)$ respondents reported following at least 100 patients in their practice. 
Table I.

Demographic Characteristics of Survey Respondents

\begin{tabular}{|c|c|}
\hline Demographic Variable & N (\% of Respondents) \\
\hline \multicolumn{2}{|l|}{ Specialty } \\
\hline Electrophysiologist & $389(90.7 \%)$ \\
\hline General cardiologist & $15(3.5 \%)$ \\
\hline Pediatric cardiologist & $8(1.9 \%)$ \\
\hline Interventional cardiologist & $4(0.9 \%)$ \\
\hline Cardiovascular surgeon & $4(0.9 \%)$ \\
\hline Other & $7(1.7 \%)$ \\
\hline \multicolumn{2}{|l|}{ Practice institution } \\
\hline University/academic center & $216(50.3 \%)$ \\
\hline Private medical practice & $115(26.8 \%)$ \\
\hline $\begin{array}{l}\text { Employed position with a } \\
\text { nonacademic medical } \\
\text { practice }\end{array}$ & $64(14.9 \%)$ \\
\hline $\begin{array}{l}\text { Public/government } \\
\text { sponsored medical center }\end{array}$ & $24(5.6 \%)$ \\
\hline Other & $8(1.9 \%)$ \\
\hline \multicolumn{2}{|l|}{ Practice setting } \\
\hline Urban & $301(70.2 \%)$ \\
\hline Suburban & $100(23.3 \%)$ \\
\hline Rural & $21(4.9 \%)$ \\
\hline \multicolumn{2}{|l|}{ Practice country } \\
\hline United States & $298(70.1 \%)$ \\
\hline Canada & $18(4.2 \%)$ \\
\hline Japan & $14(3.3 \%)$ \\
\hline United Kingdom & $11(2.6 \%)$ \\
\hline Australia & $9(2.1 \%)$ \\
\hline Brazil & $9(2.1 \%)$ \\
\hline Netherlands & $6(1.4 \%)$ \\
\hline India & $5(1.2 \%)$ \\
\hline Mexico & $5(1.2 \%)$ \\
\hline Other & $50(12 \%)$ \\
\hline \multicolumn{2}{|l|}{$\begin{array}{l}\text { Country income group by } \\
\text { place of practice }^{\dagger}\end{array}$} \\
\hline United States or Canada & $316(74.4 \%)$ \\
\hline $\begin{array}{l}\text { High-income country } \\
\text { outside of the United } \\
\text { States and Canada }\end{array}$ & $70(16.5 \%)$ \\
\hline $\begin{array}{l}\text { Lower- or upper-middle } \\
\text { income country }\end{array}$ & $39(9.2 \%)$ \\
\hline
\end{tabular}

†"High-income" countries are defined as those having per capita income of $\$ 12,616$ USD or greater; "upper-middle income" countries are defined as those having per capita income from $\$ 4,086$ to $\$ 12,615$ USD; "lower-middle income" countries are defined as those having per capita income from $\$ 1,036$ to $\$ 4,085$ USD (World Bank classifications). There were no respondents in this sample from "low-income" countries (per capita income of $\$ 1,035$ USD or less). $N=429$. Some columns may contain fewer total responses due to incomplete responses.
One hundred two $(23.8 \%)$ of those surveyed indicated that in the country where they practice, there were patients who did not receive pacemakers or ICDs due to inability to afford them. Fortyfour $(10 \%)$ respondents indicated that they were aware of some patients who, due to an inability to afford a new one, had received a resterilized CIED. Sixty $(28.4 \%)$ of those surveyed said they would be interested in receiving postmortem pacemakers or ICDs for use in their patients; $41(9.6 \%)$ had personally implanted a resterilized pacemaker or ICD, of whom $17(41.5 \%)$ practice in lowerand upper-middle income countries. Forty-five respondents reported having received donation of brand new CIEDs from Heartbeat International, Inc. (6), other charitable sources (7), or directly from the device manufacturers (32). One hundred forty-one respondents $(33.1 \%)$ reported having been asked by their patients or patients' family members about the possibility of donating their CIED in the event of death. Two hundred fiftyfive $(60.1 \%)$ indicated they would be interested in receiving more information about postmortem reuse of pacemakers and ICDs. Of those respondents asked about the greatest obstacles for their patients to undergo CIED implantation (whether of a new or reused device), 121 (56.5\%) reported cost, 15 (7.0\%)-access to implanting facilities, 15 (7.0\%)-access to implanting physicians, and 47 $(22.0 \%)$-other (answers not mutually exclusive; Table II). Respondents from lower- and uppermiddle income countries were nearly twice as likely to cite cost than other respondents $(90.3 \%$ vs $50.8 \%, \mathrm{P}<0.001)$.

\section{Opinions Regarding CIED Reuse}

HRS members' level of agreement with various statements about CIED reuse is shown in Figure 1 and Table III. The opinions of both donation and reuse were overall very positive, with the level of agreement (i.e., "strongly agree" or "agree") ranging from $81 \%(N=340$; "I would be comfortable asking my patients with an implanted pacemaker or ICD to consider donating their devices to a reutilization program upon their death") to $88 \%(N=375$; "I believe resterilization of pacemakers or ICDs with $>70 \%$ of the original battery life reclaimed from deceased patients in order to reimplant them in patients who cannot afford a new pacemaker is ethical, if it is proven to be safe"). Three hundred fiftythree $(84 \%)$ respondents agreed with the statement "If allowed by the law, I would be willing to implant postmortem pacemakers or ICDs that were resterilized and contained $>70 \%$ of original battery life in a patient who is unable to obtain a new device." When asked: "In the country 
Table II.

HRS Members' Perceived Barriers to Patients Obtaining Pacemakers and ICDs

\begin{tabular}{|c|c|c|c|c|c|}
\hline Barrier $^{\dagger}$ & $\begin{array}{c}\text { United } \\
\text { States } \\
\text { or } \\
\text { Canada } \\
\text { N (\% of } \\
\text { Respondents) }\end{array}$ & $\begin{array}{l}\text { High-Income } \\
\text { Countries Outside } \\
\text { of United States } \\
\text { and Canada } \\
\text { N (\% of } \\
\text { Respondents) }\end{array}$ & $\begin{array}{l}\text { Lower- and } \\
\text { Upper-Middle } \\
\text { Income } \\
\text { Countries } \\
\text { N (\% of } \\
\text { Respondents) }\end{array}$ & $\begin{array}{c}\text { All HRS } \\
\text { Members } \\
\text { N (\% of } \\
\text { Respondents) }\end{array}$ & $\mathbf{P}$ \\
\hline Cost & $89(56.0 \%)$ & $4(16.7 \%)$ & $28(90.3 \%)$ & $121(56.5 \%)$ & $<0.001$ \\
\hline $\begin{array}{l}\text { Lack of access to an } \\
\text { implanting facility }\end{array}$ & $8(5.0 \%)$ & $3(12.5 \%)$ & $4(12.9 \%)$ & $15(7.0 \%)$ & 0.160 \\
\hline $\begin{array}{l}\text { Lack of access to an } \\
\text { implanting physician }\end{array}$ & $11(6.9 \%)$ & $2(8.3 \%)$ & $2(6.5 \%)$ & $15(7.0 \%)$ & 0.961 \\
\hline Other & $30(18.9 \%)$ & $11(45.8 \%)$ & $6(19.4 \%)$ & $47(22.0 \%)$ & 0.012 \\
\hline None & 30 (18.9\%) & $6(25.0 \%)$ & $0(0 \%)$ & $36(16.8 \%)$ & 0.019 \\
\hline
\end{tabular}

${ }^{\dagger}$ Respondents were asked, "What are the greatest barriers your patients face in obtaining pacemakers or ICDs? Mark all that apply." Answers are not mutually exclusive. $N=214$. HRS = Heart Rhythm Society.

I believe resterilization of pacemakers or ICDs with $>70$ percent of the original battery life reclaimed from deceased patients in order to reimplant them in patients who cannot afford a new pacemaker may be safe.

I believe resterilization of pacemakers or ICDs with $>70$ percent of the original battery life reclaimed from deceased patients in order to reimplant them in patients who cannot afford a new pacemaker is ethical, if it is proven to be safe.

I believe using resterilized post mortem pacemakers or ICDs with $>70$ percent of original battery life may be a reasonable alternative to using new devices in patients who cannot afford new ones.

I would be comfortable asking my patients with an implanted pacemaker or ICD to consider donating their devices to a reutilization program upon their death.

If allowed by the law, I would be willing to implant post mortem pacemakers or ICDs that were resterilized and contained $>70$ percent of original battery life in a patient who is unable to obtain a new device.

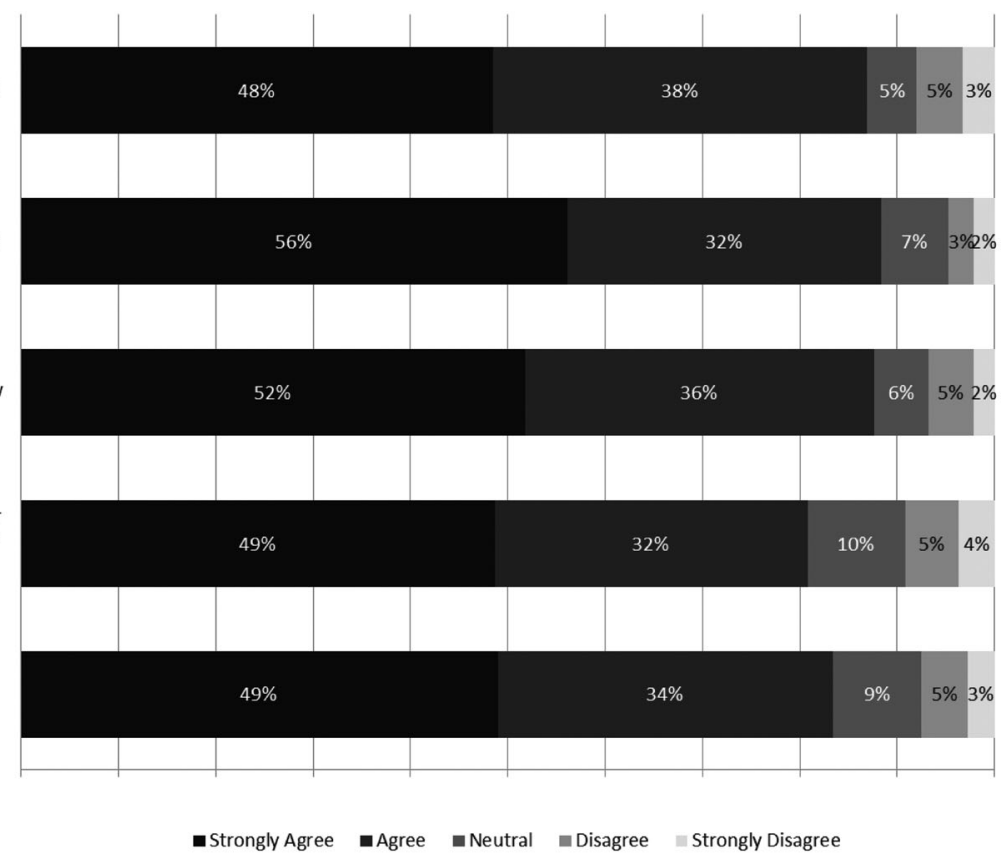

Figure 1. HRS members' agreement with specific statements regarding CIED reuse. Responses are presented as percentages of respondents $(N=429)$. CIEDs = cardiac implantable electronic devices; HRS = Heart Rhythm Society.

where I practice, most individuals needing device implantation, but who are unable to afford a new device, would be willing to undergo implantation of a device reclaimed and resterilized from a deceased person," there were 65 (31\%) "agree" or "strongly agree" responses, 94 (45\%) "neutral" responses, and 52 (24\%) "disagree" or "strongly disagree" responses.
The most commonly cited concern regarding device reuse was infection (cited by 270 respondents; $64 \%$ ), followed by device malfunction $(N$ $=125,29 \%)$, ethical concerns $(N=63,15 \%)$, "other," $(N=49,12 \%)$, and religious concerns ( $N=15,3.5 \%$; answers not mutually exclusive). Ninety-two $(22 \%)$ respondents had no concerns (Table IV). 
ELECTROPHYSIOLOGISTS' VIEWS ON CIED REUSE

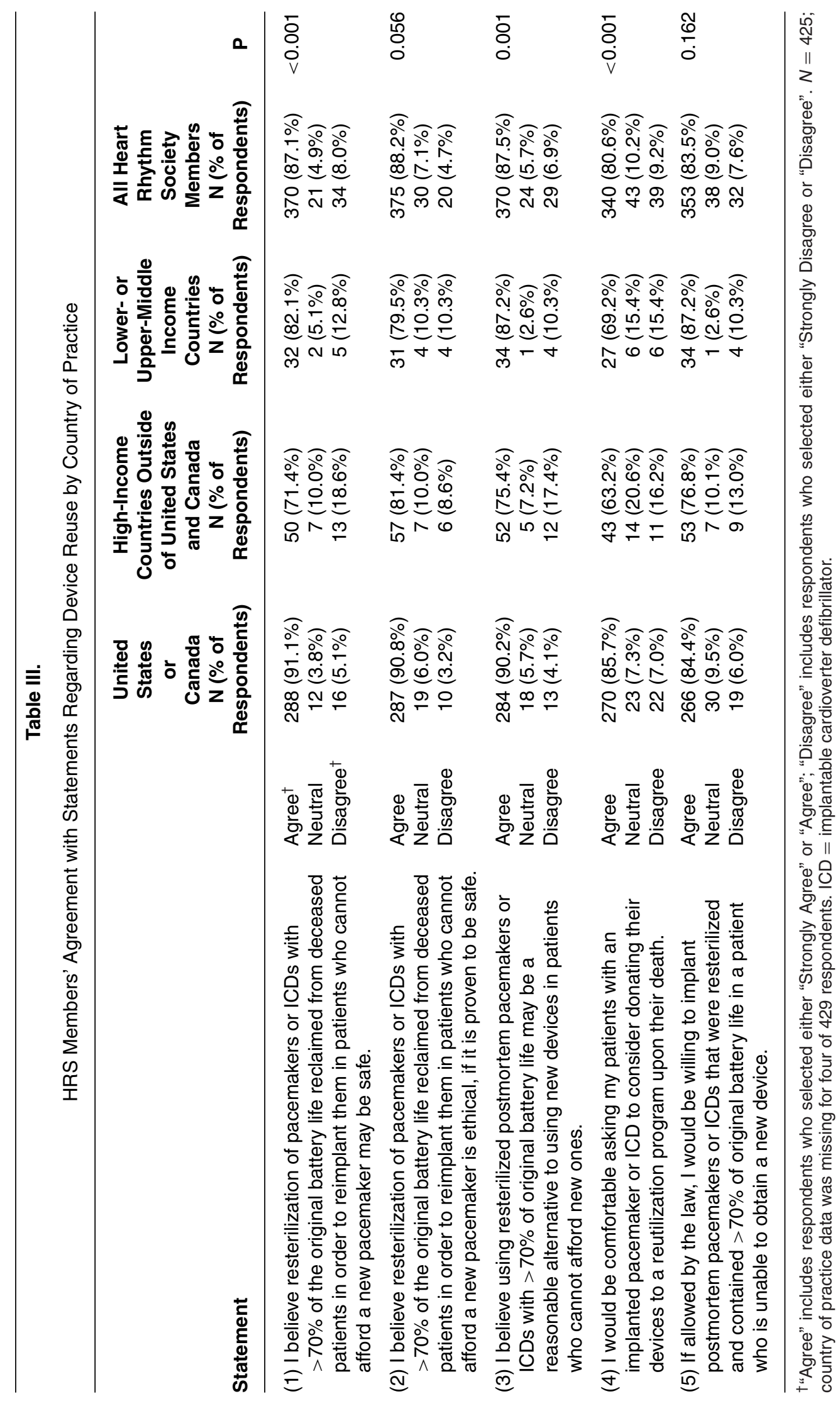


HUGHEY, ET AL.

Table IV.

HRS Members' Concerns Related to Device Reuse by Country of Practice

\begin{tabular}{|c|c|c|c|c|c|}
\hline Concern $^{\dagger}$ & $\begin{array}{c}\text { United } \\
\text { States } \\
\text { or } \\
\text { Canada } \\
\mathrm{N} \text { (\% of } \\
\text { Respondents) }\end{array}$ & $\begin{array}{c}\text { High-Income } \\
\text { Countries Outside } \\
\text { of United States } \\
\text { and Canada } \\
\mathrm{N} \text { (\% of } \\
\text { Respondents) }\end{array}$ & $\begin{array}{l}\text { Lower- and } \\
\text { Upper-Middle } \\
\text { Income } \\
\text { Countries } \\
\mathrm{N} \text { (\% of } \\
\text { Respondents) }\end{array}$ & $\begin{array}{c}\text { All HRS } \\
\text { Members } \\
\mathrm{N}(\% \text { of } \\
\text { Respondents) }\end{array}$ & $\mathbf{P}$ \\
\hline Infection & 200 (63.3\%) & $44(62.9 \%)$ & $26(66.7 \%)$ & $270(63.5 \%)$ & 0.911 \\
\hline Device malfunction & $89(28.2 \%)$ & $24(34.3 \%)$ & $12(30.8 \%)$ & $125(29.4 \%)$ & 0.585 \\
\hline Ethical & $33(10.4 \%)$ & $21(30.0 \%)$ & $9(23.1 \%)$ & $63(14.8 \%)$ & $<0.001$ \\
\hline Other & $35(11.1 \%)$ & $10(14.3 \%)$ & $4(10.3 \%)$ & 49 (11.5\%) & 0.724 \\
\hline Religious & $11(3.5 \%)$ & $4(5.7 \%)$ & $0(0 \%)$ & 15 (3.5\%) & 0.300 \\
\hline None & $73(23.1 \%)$ & $12(17.1 \%)$ & $7(17.9 \%)$ & $92(21.6 \%)$ & 0.462 \\
\hline
\end{tabular}

${ }^{\dagger}$ Respondents were asked, "What are your greatest concerns regarding the reutilization of postmortem pacemakers or ICDs? Please mark all that apply." Answers are not mutually exclusive. $N=425$; country of practice data was missing for four of 429 respondents. HRS $=$ Heart Rhythm Society.

\section{Attitudes toward CIED Reuse Across Country Income Groups}

The response rates were similar among physicians from the United States or Canada $(10 \%)$, high-income countries outside of the United States or Canada (13\%), and upper- or lower-middle income countries (13\%). In general, respondents from the United States or Canada were more likely to agree with positive statements about device reuse than respondents from other high-income countries and respondents from lower- and middle-income countries (Table III). However, when responses from the United States, Canada, and other high-income countries were grouped together and compared against those from lower- and upper-middle income countries, there were no statistically significant differences.

There were no significant differences between respondents from the United States/Canada, other high-income countries, and lower- and uppermiddle income countries in terms of naming their main concern regarding CIED reuse (infection, device malfunction, other, religious, and none); however, respondents from the United States/Canada were less likely to cite ethical concerns than the other two groups $(10 \%$ vs $30 \%$ vs $23 \%, \mathrm{P}<0.001$, see Table IV).

To determine the impact of physician immigration on the opinions of reuse, subgroup analysis was performed excluding responses from physicians who stated that they grew up in a different country than the one in which they currently practice (i.e., immigrants). Rates of agreement with each of the five positive statements regarding CIED reuse were directionally similar to the responses observed in the entire-group analysis that also included immigrant responses (United States/Canada-91.2\% vs high-income countries outside of United States/Canada-63.5\% vs lowerand upper-middle income countries-81.6\%, $\mathrm{P}<$ 0.001 for Statement 1; $90.3 \%$ vs $75.0 \%$ vs $78.9 \%$, $\mathrm{P}=0.009$ for Statement 2; $90.7 \%$ vs $66.7 \%$ vs $86.8 \%, \mathrm{P}<0.001$ for Statement $3 ; 85.3 \%$ vs $60.0 \%$ vs $71.1 \%, \mathrm{P}=0.001$ for Statement $4 ; 82.4 \%$ vs $68.6 \%$ vs $86.8 \%, \mathrm{P}=0.041$ for Statement 5 ). Similarly, the finding that respondents from the United States and Canada have lower rates of concern regarding the ethics of CIED reuse than other respondents was still the case when excluding immigrant respondents $(10.1 \%$ vs $30.8 \%$ vs $23.7 \%, \mathrm{P}<0.001)$.

\section{Discussion}

\section{Major Findings}

The main finding of this study is that a large majority of HRS survey respondents support the concept of postmortem pacemaker and ICD reuse for patients in LMICs who cannot afford new devices. This finding was consistent across the spectrum of national incomes with strong support in the United States/Canada, other high-income countries, and lower- and upper-middle income countries. There appears to be a widespread belief among respondents that reimplantation of resterilized CIEDs is safe, reasonable, and ethical. While support for CIED reuse is higher in the United States and Canada than in other highincome countries, when taken together, HRS members from these countries display an approval rate similar to respondents from lower- and uppermiddle income countries. 
Interestingly, about a third of HRS physicians surveyed reported having been asked about postmortem device donation by their patients or the patient's family. This finding is consistent with the survey data by Gakenheimer et al., indicating that $87 \%$ of patients with CIEDs and $71 \%$ of the general population would support a pacemaker reutilization initiative if given the opportunity. ${ }^{3}$ These data suggest that there is enough support for donation among the patients and their families that a large number of devices would potentially be available, if legal and regulatory issues surrounding CIED collection and reprocessing were resolved.

Chief among these regulatory and legal issues-detailed elsewhere by Kirkpatrick et al. ${ }^{5}$ is current U.S. Food and Drug Administration (FDA) policy, which designates pacemakers as "single-use device" and considers pacemaker reuse an "objectionable practice." ${ }^{6}$ While this FDA policy has not been revised since 1995, My HeartYour Heart is currently engaged with the FDA to obtain an investigational exemption detailing protocols for postmortem CIED acquisition, testing, sterilization, and delivery, for the purposes of a large clinical trial of CIED reuse. If these protocols are proven to be safe and replicable by others, they could potentially serve as a framework for future changes to FDA policy. Although local laws in recipient countries are also likely to influence the practice of CIED reuse, many LMICs currently have a less litigious climate than the United States and other potential donor countries. Since CIEDs are labeled as single-use, warranties do not cover reuse, and manufacturers would be unlikely to be held responsible in cases of reused device malfunction. ${ }^{5}$

CIEDs reuse may also conflict with the traditional practice of returning explanted devices to the original manufacturer for analysis for quality improvement testing. Postmortem property rights pertaining to explanted medical devices are not yet well established and it is unclear which legal party has jurisdiction to determine what is done with an explanted CIED. Also, a theoretical concern exists that international distribution of explanted CIEDs could inadvertently result in the creation of a "black market" for devices, particularly in LMICs where resources for market regulation are limited. ${ }^{7}$

In our study, the most prevailing concern among HRS physician members regarding device reuse was infection risk, which was reported by $64 \%$ of respondents. Concern about device malfunction was reported only by $29 \%$. Despite the inherent risk of transmitting infection from the device donor to the recipient, a meta-analysis including 18 cohort studies of pacemaker reuse showed that the risk of infection was $<2 \%$, and not statistically different from the infection risk associated with implantation of new devices (odds ratio 1.31 [0.50 to 3.40$], \mathrm{P}=0.580) .{ }^{8}$ Standardized sterilization protocols, similar to those for hemodialysis filters or other reusable medical equipment, ${ }^{5}$ are needed to ensure that sterilization is effective across multiple donation sites. For example, in one case series, debris was manually removed from the device with pipe cleaners, bathed in isopropyl alcohol, soaked overnight in an enzymatic cleaner, wiped with $70 \%$ ethanol, air dried, packed in gas-permeable envelopes, and finally decontaminated via ethylene oxide gas sterilization. ${ }^{9}$

The aforementioned meta-analysis also showed that device malfunction was more likely with reused devices (odds ratio 5.80 [1.93 to 17.47], $\mathrm{P}=0.002$ ), with malfunction occurring in $0.68 \%$ of reimplanted devices-probably an acceptable rate for a patient suffering from a bradyarrhythmia whose alternative is no device at all. Malfunctions were mostly attributed to abnormalities in set screws which may have occurred during extraction. This risk could be reduced by adequate training of funeral directors and close monitoring for device malfunction after reimplantation. ${ }^{8}$

In terms of long-term outcomes, a French study examining 151 patients who underwent implantation of a resterilized pacemaker found no differences in actuarial survival compared to those who received a new device ${ }^{10}$; similarly, a study from Sweden, where reused pacemakers accounted for $5 \%$ of all implanted devices in 1996, showed no evidence of increased patient risk associated with pacemaker reuse. ${ }^{11}$ Still, the high degree of concern for infection among HRS members in this survey highlights the need for a robust prospective clinical trial to more accurately quantify this risk before the practice of device reuse can be expanded. Efforts to recruit physicians to participate in reuse programs will need to emphasize safety.

The finding that HRS members practicing in high-income countries outside of the United States and Canada are less likely than those from the United States and Canada to presently support device reuse may have important implications, given that a significant fraction of potential donor patients reside in wealthy nations outside of North America. ${ }^{12}$ Even though nearly a third $(98,31.0 \%)$ of respondents who practice in the United States and Canada actually immigrated to these countries, these trends persisted even when excluding immigrants from the analysis. A higher rate of ethical concerns among physicians practicing in high-income countries excluding the 
United States and Canada may largely explain this discrepancy. The reasons for the higher rate of ethical concern outside of the United States and Canada are not clear from these data. Variations in ethical standards, cultural beliefs, and level of trust in the reliability of potential device reuse programs may account for some of these differences. One respondent from China cited a concern that "patients may not believe [device reuse] is safe," suggesting that physicians' anticipation of patients' concerns may also play a role in their receptiveness to device reimplantation. Further study is needed to identify the root causes of the differences in the degree of ethical concerns, if efforts to involve physicians from outside the United States and Canada in device reuse programs are to be successful. The ethical implications of CIED reuse-in particular, whether it is ethical to transfer resterilized CIEDs with possible higher risk of malfunction and less battery life than a new device to LMICshave been discussed previously by Aragam et al. ${ }^{2}$ Our position is that implantation of resterilized CIEDs is ethical because it is only offered to patients who could not otherwise obtain a device after informed consent, and it offers significant mortality and quality-of-life benefit compared to the alternative (no device). An analogous practice already commonplace in the United States is "expanded criteria donor" organ transplantation, whereby organs at greater risk of failure are offered to patients who otherwise may never receive an organ. However, rigorous quality protocols are needed to ensure that device reuse programs approximate, as much as possible, the quality of implant and follow-up care available in wealthy countries.

\section{Study Limitations}

This study has several limitations. First, because all survey respondents were HRS members and disproportionately from academic settings, their opinions are not necessarily generalizable to all electrophysiologists. However, subgroup analysis did not show any statistically significant differences in rates of agreement with positive statements regarding CIED reuse between physicians who practice in academic settings and those in private practice. While the response rate of $13 \%$ is low, it is typical for contemporary published surveys of HRS members or electrophysiologists $\left(10 \%,{ }^{13} 10 \%,{ }^{14}\right.$ and $\left.16 \%{ }^{15}\right)$. Future efforts to reach more electrophysiologists could make use of shorter surveys or monetary incentives to improve response rates. ${ }^{16}$ Since completion of the survey was voluntary, it is possible that HRS members who responded to the survey were more likely to already be familiar or interested in the concept of device reuse, and therefore their views regarding the practice may be more positive than those of all HRS physician members. Despite this possible bias, respondents to this survey were geographically well representative of HRS physician members, as $74 \%$ of all HRS physician members practice in the United States and Canada $(74 \%$ in this survey), $17 \%$ practice in highincome countries outside of the United States and Canada (14\% in this survey), and $9 \%$ practice in lower-middle income countries and upper-middle ( $8 \%$ in this survey). ${ }^{17}$ It should be noted that even countries considered by the World Bank as "upper-middle" and "lower-middle" income on a per-capita basis, such as India, ${ }^{18}$ Mexico, ${ }^{19}$ and the Philippines, ${ }^{9}$ are potential beneficiaries of CIED reuse, and their views are captured in this survey. However, physicians practicing in World Bank "low-income" countries (i.e., per capita income of \$1,035 USD or less), such as Haiti and North Korea, are not well represented in the HRS $(0.03 \%$ of physician members), and none completed this survey. A separate survey specifically targeting greater numbers of implanting physicians in recipient countries should be performed to fully explore their acceptance of reuse and identify barriers to reuse. Finally, while this quantitative survey can identify trends of opinion among HRS members, it does not explore the rationale behind those opinions. Qualitative research such as interviews or focus groups would be better suited for this purpose.

\section{Conclusions}

Pacemaker and ICD reuse has the potential to help many patients who are currently denied these therapies due to prohibitive cost. A large majority of HRS survey respondents support the concept of pacemaker and ICD reuse for patients in LMICs who cannot afford new devices. Further work is needed to more definitively demonstrate the clinical efficacy and safety of this practice, and to identify potential barriers to adoption among physicians in LMICs.

Acknowledgments: We would like to thank all HRS members who responded to this survey, as well as the Membership and Executive committees of the HRS and Mr. Jay Vegso for their support in conducting this survey. My Heart-Your Heart initiative is supported by grants from the Hewlett Foundation, the Mardigian Foundation, the University of Michigan Cardiovascular Center, and gifts from Mr. and Mrs. Sheldon Davis and Mr. and Mrs. Craig Sincock. 


\section{ELECTROPHYSIOLOGISTS’ VIEWS ON CIED REUSE}

\section{References}

1. Groeneveld PW, Matta MA, Suh JJ, Heidenreich PA, Shea JA. Costs and quality-of-life effects of implantable cardioverter-defibrillators. Am J Cardiol 2006; 98:1409-1415.

2. Aragam KG, Baman TS, Kirkpatrick JN, Goldman EB, Brown AC, Crawford T, Oral H, et al. The ethics of pacemaker reuse: Might the best be the enemy of the good? Heart 2011; 97:2005-2006.

3. Gakenheimer L, Lange DC, Romero J, Kirkpatrick JN, Sovitch $\mathrm{P}$, Oral H, Eagle KA, et al. Societal views of pacemaker reutilization for those with untreated symptomatic bradycardia in underserved nations. J Interv Card Electrophysiol 2011; 30:261266.

4. The World Bank. Country and Lending Groups. Available at: http://data.worldbank.org/about/country-classifications/countryand-lending-groups. Accessed Oct 2013.

5. Kirkpatrick JN, Papini C, Baman TS, Kota K, Eagle KA, Verdino RJ, Caplan AL. Reuse of pacemakers and defibrillators in developing countries: Logistical, legal, and ethical barriers and solutions. Heart Rhythm 2010; 7:1623-1627.

6. FDA Compliance Policy Guides, CPG Sec. 310.100 Pacemaker Reuse: http://www.fda.gov/ICECI/ComplianceManuals/Compliance PolicyGuidanceManual/ucm073891.htm (accessed November 2013).

7. Baman TS, Eagle KA. Cardiac device reutilization: Is it time to "go green" in underserved countries? Pacing Clin Electrophysiol 2011; 34:651-652.

8. Baman TS, Meier P, Romero J, Gakenheimer L, Kirkpatrick JN, Sovitch P, Oral H, et al. Safety of pacemaker reuse: A metaanalysis with implications for underserved nations. Circ Arrhythm Electrophysiol 2011; 4:318-323.

9. Baman TS, Romero A, Kirkpatrick JN, Romero J, Lange DC, Sison EO, Tangco RV, et al. Safety and efficacy of pacemaker reuse in underdeveloped nations: A case series. J Am Coll Cardiol 2009; 54:1557-1558.
10. Mugica J, Duconge R, Henry L. Survival and mortality in 3,701 pacemaker patients: Arguments in favor of pacemaker reuse. Pacing Clin Electrophysiol 1986; 9:1282-1287.

11. Ryden L. Re-use of devices in cardiology. Proceedings from a Policy Conference at the European Heart House, 5-6 February, 1998. Eur Heart J 1998; 19:1628-1631.

12. Mond HG, Proclemer A. The 11th world survey of cardiac pacing and implantable cardioverter-defibrillators: Calendar year 2009-a World Society of Arrhythmia's project. Pacing Clin Electrophysiol 2011; 34:1013-1027.

13. Sears SF, Todaro JF, Urizar G, Lewis TS, Sirois B, Wallace R, Sotile W, et al. Assessing the psychosocial impact of the ICD: A national survey of implantable cardioverter defibrillator health care providers. Pacing Clin Electrophysiol 2000; 23:939-945.

14. Deering TF, Clair WK, Delaughter MC, Fisher WG, Garlitski AC, Wilkoff BL, Gillis AM. A Heart Rhythm Society Electrophysiology Workforce study: Current survey analysis of physician workforce trends. Heart Rhythm 2010; 7:1346-1355.

15. Xu W, Moore HJ, Karasik PE, Franz MR, Singh SN, Fletcher RD. Management strategies when implanted cardioverter defibrillator leads fail: Survey findings. Pacing Clin Electrophysiol 2009; 32:1130-1141.

16. Kellerman SE, Herold J. Physician response to surveys. A review of the literature. Am J Prev Med 2001; 20:61-67.

17. Vegso J, Membership Director, HRS, October 29, 2013, Personal Communication.

18. Pavri BB, Lokhandwala Y, Kulkarni GV, Shah M, Kantharia BK, Mascarenhas DA. Reuse of explanted, resterilized implantable cardioverter-defibrillators: A cohort study. Ann Intern Med. 2012; 157:542-548.

19. Nava S, Morales JL, Marquez MF, Barrera F, Gomez J, Colin L, Brugada J, et al. Reuse of pacemakers: Comparison of short and long-term performance. Circulation. 2013; 127:1177-1183.

\section{Supporting Information}

Additional Supporting Information may be found in the online version of this article at the publisher's website:

Appendix S1, Table A1: Selected studies of CIED reuse. Appendix S2: Survey Instrument. 